\title{
The languages of bacteria
}

\author{
Stephan Schauder and Bonnie L. Bassler ${ }^{1}$ \\ Department of Molecular Biology, Princeton University, Princeton, New Jersey 08544-1014, USA
}

Bacteria communicate with one another using chemical signaling molecules as words. Specifically, they release, detect, and respond to the accumulation of these molecules, which are called autoinducers. Detection of autoinducers allows bacteria to distinguish between low and high cell population density, and to control gene expression in response to changes in cell number. This process, termed quorum sensing, allows a population of bacteria to coordinately control the gene expression of the entire community. Quorum sensing confuses the distinction between prokaryotes and eukaryotes because it allows bacteria to behave as multicellular organisms, and to reap benefits that would be unattainable to them as individuals. Many bacterial behaviors are regulated by quorum sensing, including symbiosis, virulence, antibiotic production, and biofilm formation. Recent studies show that highly specific as well as universal quorum sensing languages exist which enable bacteria to communicate within and between species. Finally, both prokaryotic and eukaryotic mechanisms that interfere with bacterial quorum sensing have evolved. Specifically, the secretion of enzymes that destroy the autoinducers, and the production of autoinducer antagonists, are used by competitor bacteria and susceptible eukaryotic hosts to render quorum sensing bacteria mute and deaf, respectively. Analogous synthetic strategies are now being explored for the development of novel antimicrobial therapies.

Bacteria in communities convey their presence to one another by releasing and responding to the accumulation of chemical signaling molecules called autoinducers. This process of intercellular communication, called quorum sensing, was first described in the bioluminescent marine bacterium Vibrio fischeri (Hastings and Nealson 1977; Nealson and Hastings 1979). V. fischeri lives in symbiotic associations with a number of marine animal hosts. In these partnerships, the host uses the light produced by $V$. fischeri for specific purposes such as

${ }^{1}$ Corresponding author.

E-MAIL bbassler@molbio.princeton.edu; FAX (609) 258-6175.

Article and publication are at http://www.genesdev.org/cgi/doi/10.1101/ gad.899601. attracting prey, avoiding predators, or finding a mate. In exchange for the light it provides, $V$. fischeri obtains a nutrient-rich environment in which to reside (Ruby 1996; Visick and McFall-Ngai 2000). A luciferase enzyme complex is responsible for light production in $V$. fischeri. Bioluminescence only occurs when $V$. fischeri is at high cell number, and this process is controlled by quorum sensing. Specifically, the production and accumulation of, and the response to, a minimum threshold concentration of an acylated homoserine lactone (HSL) autoinducer regulates density-dependent light production in $V$. fischeri, and enables $V$. fischeri to emit light only inside the specialized light organ of the host but not when free-living in the ocean. The reason for this is twofold. First, only under the nutrient-rich conditions of the light organ can $V$. fischeri grow to high population densities, and second, trapping of the diffusible autoinducer molecule in the light organ with the bacterial cells allows it to accumulate to a sufficient concentration that $V$. fischeri can detect it.

Engebrecht and Silverman discovered the regulatory circuit controlling quorum sensing in $V$. fischeri (Engebrecht et al. 1983; Engebrecht and Silverman 1984, 1987). They showed that two regulatory components are required for the process. The LuxI protein is responsible for production of the HSL autoinducer, and the LuxR protein is responsible for binding the HSL autoinducer and activating transcription of the luciferase structural operon at high cell density (Engebrecht et al. 1983; Engebrecht and Silverman 1984). They showed that, as an autoinducer-producing population of $V$. fischeri cells grows, the concentration of autoinducer increases as a function of increasing cell-population density. When the autoinducer concentration reaches the micromolar range, it can interact with the LuxR protein, and the LuxR-autoinducer complex binds the luciferase promoter to activate transcription. Therefore, this quorum sensing circuit allows light production to be tightly correlated with the cell population density. For over 10 years the $V$. fischeri LuxI/LuxR signal-response system was considered a curious, but isolated, example of bacterial communication that had presumably evolved for a specific purpose required for the colonization of a symbiotic host. However, we now understand that most bac- 
teria communicate using secreted signal molecules to control the behavior of the group. We know that a vast array of molecules are used as the signals, that individual species of bacteria simultaneously produce, detect, and respond to multiple classes of chemical signals, and finally, that the signal-detection apparatuses are highly varied and appear precisely tuned for optimized communication in specialized niches. These findings indicate that quorum sensing enables bacteria to talk to each other, and in many cases, to be multilingual.

\section{Gram-negative bacteria use homoserine lactones as words}

Communication via LuxI/LuxR (HSL/transcriptional activator) signaling circuits appears to be the standard mechanism by which Gram-negative bacteria talk to each other, as quorum sensing systems resembling the canonical $V$. fischeri circuit have been shown to control gene expression in over 25 species of Gram-negative bacteria (Fuqua et al. 1996; Bassler 1999; de Kievit and Iglewski 2000; Miller and Bassler 2001). In every case, an acylated HSL is the signal molecule whose synthesis is dependent on a LuxI-like protein. A cognate LuxR-like protein is responsible for recognition of the HSL autoinducer and subsequent transcriptional activation of downstream target genes. A general model showing the fundamental components of a Gram-negative quorum sensing circuit is presented in Figure 1. Many physiologi-



Figure 1. The LuxI/LuxR quorum sensing system of Gramnegative bacteria. Two regulatory proteins control quorum sensing in most Gram-negative bacteria. The LuxI-like proteins are the autoinducer synthases, and they catalyze the formation of a specific acyl-HSL autoinducer molecule (green pentagons). The autoinducer freely diffuses through the cell membrane and accumulates at high cell density. At high autoinducer concentration, the LuxR-like proteins bind their cognate autoinducers. The LuxR-autoinducer complexes bind at target gene promoters and activate transcription. cal processes are regulated by these cell-cell communication systems, including virulence, biofilm formation, antibiotic production, and conjugation.

In all cases, the biochemical mechanism of action of the LuxI/LuxR pairs is conserved. The LuxI-like enzymes produce a specific acylated HSL by coupling the acyl-side chain of a specific acyl-acyl carrier protein (acyl-ACP) from the fatty acid biosynthetic machinery to the homocysteine moiety of $S$-adenosylmethionine (SAM). The ligated intermediate lactonizes to form the acyl-HSL, and methylthioadenosine (MTA) is released (Hanzelka and Greenberg 1996; More et al. 1996; Val and Cronan 1998; Parsek et al. 1999). Some representative acyl-HSL autoinducers are shown in Figure 2A.

Acyl-HSL autoinducers freely diffuse through the bacterial membrane, allowing them to increase in concentration in the external environment in conjunction with cell population growth (Kaplan and Greenberg 1985). The LuxR-like proteins function by binding their partner autoinducers and activating transcription of target DNA. The amino-terminal regions of the LuxR-like proteins are involved in HSL binding, and the C-terminal domains of the proteins are responsible for oligomerization and promoter DNA binding. A similar promoter element is bound by each of the LuxR-type proteins (Slock et al. 1990; Choi and Greenberg 1991, 1992; Stevens et al. 1994; Poellinger et al. 1995; Stevens and Greenberg 1997; Stevens et al. 1999).

Rather exquisite signaling specificity exists in LuxI/ LuxR-type circuits (Gray et al. 1994). This facet of the systems is astonishing considering the remarkable similarity of both the HSL signal molecules and the LuxRlike proteins. Presumably, the specificity inherent in these systems stems from a high selectivity of the LuxR proteins for a cognate HSL ligand. Similarly, the specificity in signal production must come from a precise interaction of each LuxI-type protein with a particular acyl-ACP.

In many instances, further regulatory complexity has been added to the basic LuxI/LuxR signal-response circuit. For example, the opportunistic human pathogen Pseudomonas aeruginosa uses a hierarchical quorum sensing circuit to regulate expression of virulence factors and biofilm formation (Parsek and Greenberg 1999; de Kievit and Iglewski 2000). In P. aeruginosa, two LuxI/ LuxR pairs exist (called LasI/LasR and RhlI/RhlR), and they function in tandem to control the target outputs (Passador et al. 1993; Brint and Ohman 1995). Specifically, the LasI/LasR system initiates the signaling cascade by inducing the transcription of virulence factors at high cell density (Jones et al. 1993; Passador et al. 1993; Davies et al. 1998). Additionally, LasI/LasR activates the expression of rhlI and rhlR (Ochsner and Reiser 1995). RhlI/RhlR function to further activate genes that are already under LasI/LasR control, and RhlI/RhlR also activate additional specific target genes (Brint and Ohman 1995; Latifi et al. 1996; Pearson et al. 1997; Pesci et al. 1997; Hassett et al. 1999; Parsek and Greenberg 1999; Whiteley et al. 1999). Regulation of RhlI/RhlR by LasI/ LasR ensures that the establishment of the two quorum 


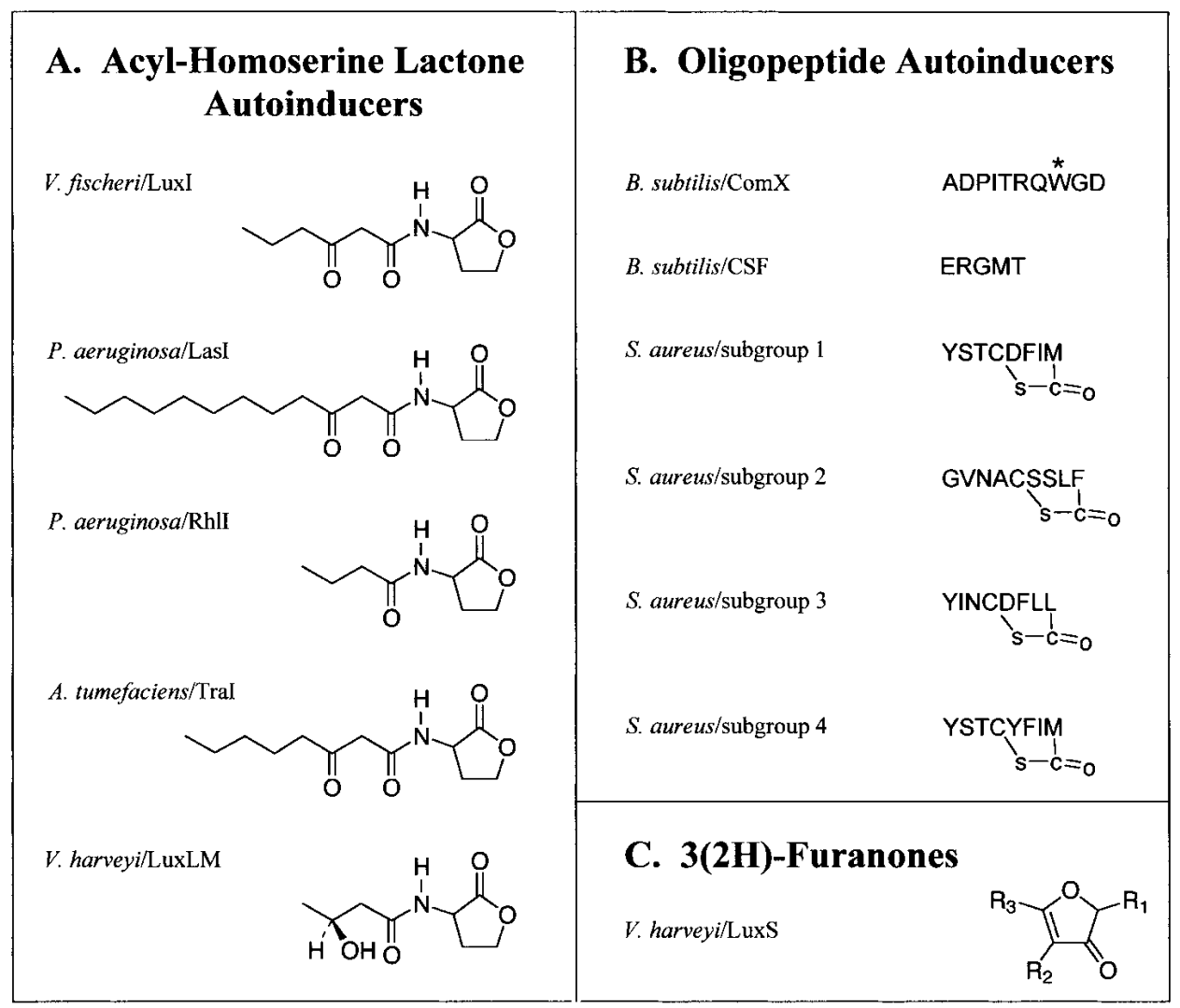

Figure 2. Different classes of autoinducers. The figure shows three classes of molecules used as autoinducers. $(A)$ Representative acyl-homoserine lactone autoinducers used by Gram-negative bacteria. (B) Representative oligopeptide autoinducers used by Grampositive bacteria. The ${ }^{*}$ on the $B$. subtilis ComX oligopeptide autoinducer represents a modification of unknown structure. $(C)$ The general structure of the $3(2 \mathrm{H})$ class of furanones. A $3(2 \mathrm{H})$-furanone is suspected to be the LuxS-dependent autoinducer called AI- 2 .

sensing circuits occurs sequentially and in the correct order. A quinolone signal (PQS for Pseudomonas quinolone signal) is also involved in the $P$. aeruginosa quorum sensing regulatory hierarchy. PQS acts as another regulatory link between the LasI/LasR and RhlI/RhlR circuits (Pesci et al. 1999). Recently, a third LuxR homolog named QscR was identified from the completed genome sequence of $P$. aeruginosa. There is no indication of a cognate LuxI-like synthase that could be responsible for production of an autoinducer to which QscR responds. The function of QscR is to regulate production of the LasI-directed acyl-HSL autoinducer (Chugani et al. 2001). Apparently this complex interconnected network is responsible for precise timing of the expression of the various quorum sensing controlled target genes in $P$. aeruginosa.

In Agrobacterium tumefaciens, a pathogen that causes crown gall tumors in plants, quorum sensing controls the conjugation of a virulence plasmid called the Ti plasmid (Piper et al. 1993; Sheng and Citovsky 1996; Christie 1997). A. tumefaciens, like $V$. fischeri, employs a LuxI/ LuxR acyl-HSL/transcriptional activator system as the foundation of the quorum sensing cascade. In this case, the regulatory proteins are called TraI/TraR, and the circuit induces the expression of genes required for mating between bacterial cells and for mobilization of the Ti plasmid (Piper et al. 1993; Zhang et al. 1993; Fuqua and Winans 1994; Hwang et al. 1994). However, the A. tumefaciens circuit is also responsive to signals produced by the plant. Plant chemicals called opines produced at the site of the bacterial infection initiate the quorum sensing cascade by either inducing an activator or inhibiting a repressor of the expression of traR. The mechanism of opine regulation (i.e., activation or repression) depends on the type of Ti plasmid carried by the invading A. tumefaciens strain (Beck von Bodman et al. 1992; Dessaux et al. 1992; Fuqua and Winans 1996). This step allows the typical LuxI/LuxR-type autoinduction circuit to be established only at the bacterial-plant interface. An additional target of the TraI/TraR system is called traM. TraM down-regulates quorum sensing by binding and inhibiting TraR (Fuqua et al. 1995; Luo et al. 2000).

Many other examples of Gram-negative circuits exist that utilize a basic LuxI/LuxR quorum sensing mechanism onto which additional regulatory factors have been layered (Miller and Bassler 2001). These "designer" regulatory components enable a wide assortment of behaviors to be controlled by a common mechanism, while precisely adapting each quorum sensing circuit to the specialized needs of a particular species of bacteria residing in a unique niche. 


\section{Gram-positive bacteria speak with oligopeptides}

Quorum sensing in Gram-positive bacteria is also responsible for the control of a wide variety of functions (Grossman 1995; Lazazzera and Grossman 1998; Novick 1999). However, Gram-positive bacteria have evolved a basic communication mechanism that is different from that used by Gram-negative bacteria. In this case, the signals are modified oligopeptides that are secreted into the medium and accumulate at high cell density. The detectors for the oligopeptide signals are two-component adaptive response proteins. Bacteria use two-component proteins to detect fluctuations in environmental stimuli and relay the information regarding these changes into the cell. The mechanism of signal transduction is via a conserved phosphorylation/dephosphorylation mechanism. Analogous to Gram-negative quorum sensing bacteria, Gram-positive bacteria employ a conserved signalresponse mechanism as the foundation of the quorum sensing process, and the addition of diverse regulatory components fine-tunes each circuit to the individualized needs of the species.

A model showing the general components of a Grampositive quorum sensing circuit is presented in Figure 3. A precursor peptide is synthesized, and subsequently processed and modified to make the mature oligopeptide autoinducer molecule. This processed peptide is secreted via an ATP-Binding Cassette (ABC) transporter complex. The concentration of external signal increases as the cells grow in number. At a critical concentration of oligopeptide autoinducer, the two-component sensory recognition apparatus detects the signal. Sensory information is relayed into the cell by phosphorylation, and culminates in an appropriate alteration in gene expression (Kleerebezem et al. 1997). The detector for the oligopeptide autoinducer is called the sensor kinase, and it transfers the phosphoryl signal to a downstream regulatory component, called the response regulator. The response regulator is a DNA binding protein whose function is typically to induce the expression of density-controlled target genes (Hoch 1995). Many Gram-positive quorum sensing systems are currently under study. Here we present an outline of the systems used by Bacillus subtilis and Staphylococcus aureus, with an emphasis on the common and unique features of the two circuits. The peptide autoinducers used by these two species are shown in Figure 2B.

B. subtilis is a soil organism that uses quorum sensing to alternate between competence for DNA uptake and sporulation (Grossman 1995; Lazazzera and Grossman 1998). B. subtilis takes up DNA at the transition from exponential to stationary phase growth. This DNA is presumably used as a repository of genetic material for use in repairing damaged chromosomes. Sporulation occurs when nutrients become limiting. Two oligopeptide autoinducers regulate these lifestyle changes. In the competence process, extracellular accumulation of the processed ComX peptide autoinducer activates the ComP/ComA two-component phosphorylation cascade. The function of ComP/ComA is to increase the level of

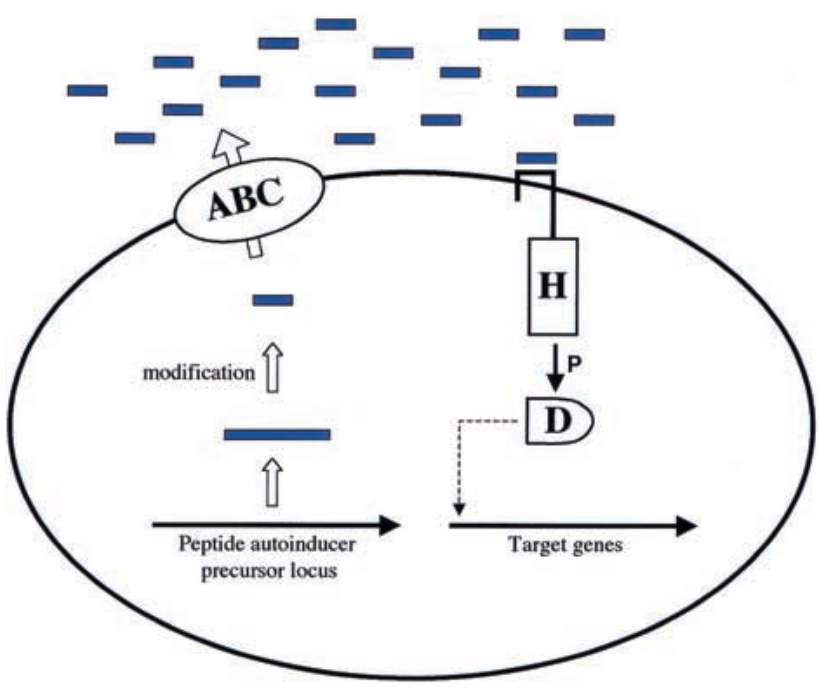

Figure 3. Oligopeptide mediated quorum sensing in Grampositive bacteria. A generalized model encompassing the components used in Gram-positive bacterial quorum sensing is shown. A specific precursor peptide (large blue bars) is produced. The precursor peptide is modified, processed, and an ATP-binding cassette $(\mathrm{ABC})$ exporter complex secretes the mature oligopeptide autoinducer (short blue bars). The oligopeptide autoinducer accumulates as the cells grow. At high cell density, the autoinducer is detected by a two-component signal transduction system. Specifically, the sensor kinase protein recognizes the autoinducer and subsequently autophosphorylates at a conserved histidine residue $(\mathrm{H})$. The phosphoryl group is transferred to a cognate response regulator protein, and this protein is phosphorylated on a conserved aspartate residue (D). The phosphorylated response regulator binds to specific target promoters to modulate the expression of quorum sensing regulated genes. $\mathrm{P}$ denotes that the mechanism of signal transduction is by phosphate transfer between the regulatory elements.

a transcriptional activator called ComK. ComK activates the expression of genes required to develop the competent state (Magnuson et al. 1994; Solomon et al. 1995, 1996; van Sinderen et al. 1995; Turgay et al. 1997, 1998). The second peptide autoinducer CSF (competence and sporulation factor) plays a role in both the competence and the sporulation processes (Solomon et al. 1996). Similarly to other autoinducers, CSF increases in concentration in the extracellular environment as a function of cell population density. However, in contrast to other oligopeptide autoinducers, CSF has an intracellular signaling role. CSF is imported into B. subtilis (Solomon et al. 1995; Lazazzera et al. 1997). At low internal CSF concentrations, the peptide promotes development of the competent state by indirectly increasing the activity of ComA, the two-component protein required for competence (Solomon et al. 1996; Perego 1997). However, high internal CSF concentrations inhibit competence and drive $B$. subtilis into the sporulation process. In this case, CSF indirectly increases the activity of a two-component protein called SpoOA, which is required for sporulation (Perego et al. 1994; Grossman 1995; Solomon et al. 1995). Precise modulation of the internal concentration 
of CSF likely shifts the equilibrium toward the lifestyle that is most appropriate for a given set of environmental circumstances.

In the invasive pathogen $S$. aureus, a quorum sensing system called agr regulates virulence. Truncation of a 46-amino acid precursor peptide, and subsequent introduction of a thiolactone ring yields the mature autoinducing oligopeptide (Ji et al. 1995, 1997). The processed oligopeptide is secreted, and subsequently increases to a sufficient concentration for detection by the AgrC/AgrA sensor kinase/response regulator pair (Morfeldt et al. 1988; Peng et al. 1988; Novick et al. 1995). PhosphoAgrA increases the production of an mRNA effector molecule called RNAIII. RNAIII, by an unknown mechanism, positively influences the expression of several secreted pathogenicity determinants (Morfeldt et al. 1995). $S$. aureus strains can be classified into four different groups based on the specificity of their autoinducer oligopeptides. A fascinating aspect of the $S$. aureus system is that each oligopeptide autoinducer activates its group's specific agr virulence system but inhibits the $a g r$ systems of the other S. aureus groups (Ji et al. 1997; Mayville et al. 1999; Otto et al. 1999). Apparently, during host invasion, the $S$. aureus group that establishes its quorum sensing cascade fastest eliminates the possibility of successful invasion by a competing group of $S$. aureus.

\section{Multilingual bacteria: the universal LuxS language}

All of the quorum sensing systems we have described so far rely on the precise recognition of an autoinducer by its cognate detector. The tight specificity inherent in these communication systems is presumably required to prevent the bacteria from being confused by noise. Further, it allows them to keep their conversations private, i.e., within their own species. However, recent studies suggest that bacteria may have evolved multiple languages that serve different purposes. It appears that many bacteria possess a species-specific language as well as a species-nonspecific language. These findings imply that bacteria can assess their own population numbers and also the population density of other species of bacteria in the vicinity. Furthermore, distinct responses to the intraspecies and interspecies signals allow a particular species of bacteria to properly modulate its behavior depending on whether it makes up a majority or a minority of any given consortium.

Studies of interspecies communication originated with the bioluminescent marine bacterium Vibrio harveyi (Bassler et al. 1997). V. harveyi, while closely related to $V$. fischeri, does not live in symbiotic associations with higher organisms. Rather, $V$. harveyi is found freeliving in the seawater, in shallow sediments and on the surfaces and in the gut tracts of various marine animals. Similar to $V$. fischeri, $V$. harveyi uses quorum sensing to control bioluminescence. However, unlike $V$. fischeri and all other Gram-negative quorum sensing bacteria, $V$. harveyi does not employ a canonical LuxI/
LuxR-type quorum sensing mechanism (Bassler et al. 1993, 1994a,b).

$V$. harveyi has evolved a quorum sensing circuit that has characteristics typical of both Gram-negative and Gram-positive bacterial quorum sensing systems. Specifically, $V$. harveyi uses an acyl-HSL autoinducer similar to other Gram-negative quorum sensing bacteria, but the signal detection and relay apparatus consists of twocomponent proteins similar to the quorum sensing systems of Gram-positive bacteria. In addition, $V$. harveyi produces and responds to a novel quorum sensing autoinducer that appears to be designed for interspecies cellcell communication.

A model showing the hybrid quorum sensing circuit employed by $V$. harveyi is presented in Figure 4. V. harveyi produces two autoinducers termed AI-1 and AI-2 (Bassler et al. 1993, 1994a). AI-1 is an acyl-HSL, (Cao and Meighen 1989) but its synthesis is not dependent on a $V$. fischeri-like LuxI function. The luxLM locus is required for production of AI-1 (Bassler et al. 1993). LuxLM does not share any homology with the LuxI family of autoinducer synthases; (Bassler et al. 1993) however, the same

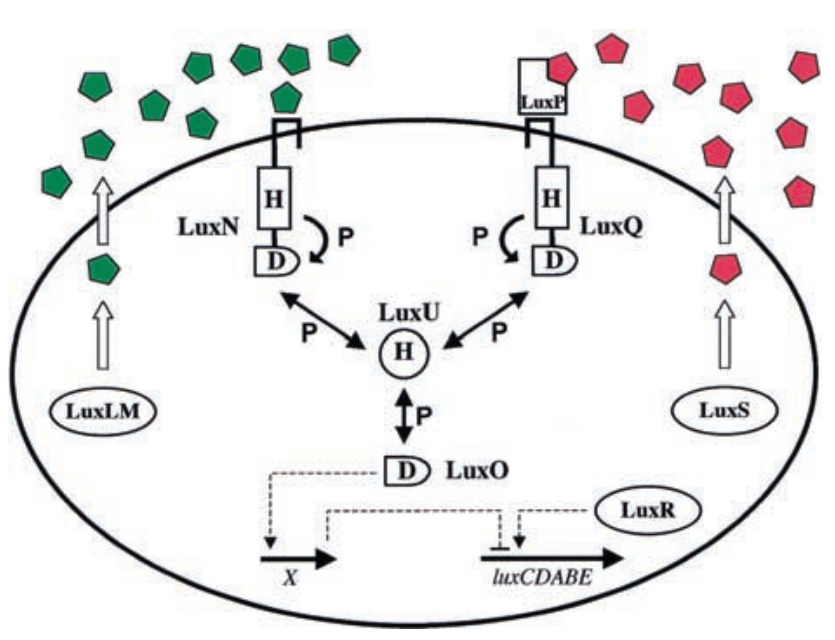

Figure 4. The hybrid quorum sensing circuit of $V$. harveyi. Elements characteristic of both Gram-negative and Gram-positive bacterial quorum sensing systems are combined to make up the $V$. harveyi circuit. An acyl-HSL autoinducer (AI-1, green pentagons) is produced by the activity of LuxLM. A second autoinducer (AI-2, red pentagons) is synthesized by the enzyme LuxS. AI- 2 is proposed to be a furanone. Both autoinducers accumulate as a function of cell density. The sensor for AI- 1 is LuxN, and two proteins, LuxP and LuxQ, function together to detect AI-2. LuxP is homologous to the periplasmic ribose binding protein of E. coli. LuxN and LuxQ are hybrid sensor kinase/ response regulator proteins that transduce information to a shared integrator protein called LuxU. LuxU sends the signal to the response regulator protein LuxO. The mechanism of signal transduction is phosphorelay (denoted $\mathrm{P}$ ). LuxO controls the transcription of a putative repressor protein (denoted X), and a transcriptional activator protein called LuxR is also required for expression of the luciferase structural operon (luxCDABE). The details of the signaling mechanism are given in the text. The conserved phosphorylation sites on the two-component proteins are indicated as $\mathrm{H}$ (histidine) and $\mathrm{D}$ (aspartate). 
enzymatic mechanism is probably used to make the $V$. harveyi acyl-HSL from SAM and a specific acyl-ACP (Hanzelka et al. 1999). The second autoinducer, AI-2, is not an HSL. Recent studies suggest that AI-2 is a furanone (Schauder et al. 2001). Its synthesis is dependent on the LuxS protein (Surette et al. 1999). The biosynthesis and structure of AI-2 are discussed below. The $V$. harveyi acyl-HSL autoinducer is shown in Figure 2A, and the general structure of a furanone is shown in Figure 2C.

As depicted in Figure 4, the cognate sensors LuxN and LuxPQ recognize AI-1 and AI-2, respectively. LuxP is a soluble periplasmic protein that is homologous to the ribose binding protein of Escherichia coli and Salmonella typhimurium. LuxP is proposed to be the primary receptor for AI-2. LuxP, in complex with AI-2, interacts with LuxQ to send the AI-2 signal (Bassler et al. 1993, 1994a). LuxN and LuxQ are two component hybrid sensor kinase/response regulator proteins that funnel their phosphorylation signals to a shared integrator protein called LuxU (Freeman and Bassler 1999b). LuxU channels the signal to a final response regulator protein called LuxO (Bassler et al. 1994b; Freeman and Bassler 1999a). LuxO is a $\sigma^{54}$-dependent transcriptional activator whose hypothesized role is to induce the expression of a repressor of the luciferase structural operon (IuxCDABE) (Lilley and Bassler 2000). This putative repressor is called $\mathrm{X}$ in Figure 4. A transcriptional activator called LuxR is also required for expression of IuxCDABE (Martin et al. 1989; Showalter et al. 1990). However, the $V$. harveyi LuxR is not similar to the LuxR from $V$. fischeri and other Gram-negative quorum sensing bacteria.

The $V$. harveyi quorum sensing circuit is proposed to function as follows. At low cell density, in the absence of AI-1 and AI-2, LuxN and LuxQ are autophosphorylating kinases that transfer phosphate to LuxU. LuxU subsequently transfers the phosphate to LuxO. Phospho-LuxO is active (Freeman and Bassler 1999a,b; Freeman et al. 2000). In conjunction with the alternative sigma factor $\sigma^{54}$, phospho-LuxO activates the transcription of $\mathrm{X}$, and $\mathrm{X}$, in turn, represses the expression of luxCDABE (Lilley and Bassler 2000). Therefore, under the low cell density condition $V$. harveyi makes no light. At high cell density, when the autoinducers have accumulated, interaction of AI-1 and AI-2 with LuxN and LuxPQ induces LuxN and LuxQ to switch from kinase mode to phosphatase mode. As phosphatases, LuxN and LuxQ drain phosphate from LuxO via LuxU. Dephospho-LuxO is inactive (Freeman and Bassler 1999a,b; Freeman et al. 2000). Thus, the repressor $X$ is not transcribed, and the LuxR protein activates transcription of $\operatorname{lux} C D A B E$. Therefore, under the high cell density condition $V$. harveyi emits light.

The benefit $V$. harveyi derives from having two redundant quorum sensing systems was a mystery. To examine the specific function of each system, $V$. harveyi reporter strains capable of exclusive response to AI-1 or AI-2 were constructed. These reporters were used to demonstrate that many species of bacteria produce AI-2 activity, but only very rarely could a species of bacteria be identified that produced an AI-1 activity (Bassler et al. 1997; Surette and Bassler 1998). These experiments led to the hypothesis that $V$. harveyi uses AI-1 for intraspecies communication and AI-2 for interspecies cellcell signaling (Bassler et al. 1997; Bassler 1999). Unlike $V$. fischeri that uses quorum sensing in a pure culture in a specialized host light organ, $V$. harveyi uses quorum sensing in environments containing other species of bacteria. It appears that a single species-specific language is not sufficient for density sensing in a bacterium that usually lives in mixed-species consortia. $V$. harveyi could use the highly specific AI-1 language to assess its own population density, and use the nonspecific AI-2 language to assess the population density of all the other species in the vicinity. Consistent with this idea, it has now been shown that $V$. harveyi possesses genes that are regulated by both autoinducers and also genes that are only controlled by AI-1 or AI-2. Presumably, these three classes of genes allow $V$. harveyi to differentially regulate behavior based on whether it or some other species of bacteria predominates (K. Mok, J. Henke, and B. Bassler, unpubl.).

Additional evidence for the universality of the AI-2 language came with the cloning of $\operatorname{lux} S$, the function required for production of AI-2 in $V$. harveyi (Surette et al. 1999). Database analysis showed that conserved LuxS homologs exist in over 30 species of bacteria (Surette et al. 1999; Miller and Bassler 2001). These species include but are not restricted to: E. coli, S. typhimurium, Salmonella typhi, Salmonella paratyphi, Shigella flexneri, Haemophilus influenzae, Helicobacter pylori, B. subtilis, Borrelia burgdorferi, Neisseria meningitidis, Yersinia pestis, Campylobacter jejuni, Vibrio cholerae, Vibrio vulnificus, Mycobacterium tuberculosis, Enterococcus faecalis, S. pneumoniae, Streptococcus pyogenes, S. aureus, Clostridium perfringens, Clostridium difficile, and Klebsiella pneumoniae. Most of these bacteria have been shown to produce AI-2, and mutants have been made in E. coli, S. typhimurium, $H$. pylori, $S$. flexneri, V. cholerae, V. vulnificus, and S. aureus. In each case, mutagenesis of luxS eliminated AI-2 production (Sperandio et al. 1999; Joyce et al. 2000; Kim et al. 2000; Lyon et al. 2000; Day and Maurelli 2001). These results show that LuxS defines a new bacterial language. What is remarkable about this list is that it includes both Gramnegative and Gram-positive bacterial species, indicating that AI-2 signaling is widely used.

Similar to $V$. harveyi, many of the other bacteria that have luxS and produce AI-2 also have another quorum sensing circuit (for example, B. subtilis and S. aureus). This fact raises the possibility that numerous bacteria use quorum sensing languages in a manner similar to $V$. harveyi. In general, bacteria may have a language that is designed exclusively for intraspecies communication, as well as AI-2 (or another language) that is used for interspecies communication. Multilingual capabilities could enhance survival and interaction in natural habitats in which bacteria exist in mixed-species communities.

It is of interest to know what genes are regulated by 
AI-2 in the different luxS-containing bacteria. There are reports indicating that virulence is regulated by AI-2 in E. coli, S. flexneri, S. aureus, and V. vulnificus (Sperandio et al. 1999; Kim et al. 2000; Lyon et al. 2000; Day and Maurelli 2001). However, not all of the bacteria that have IuxS are pathogens. It is suspected that a fundamental regulon of genes is controlled by AI- 2 in all luxScontaining bacteria. In addition, niche-specific genes will be added to the regulon, and these genes will differ for each LuxS-containing species of bacteria. Presumably, these species-specific targets will encode functions whose expressions are beneficial only when a particular species of bacteria exists in consortia. It already appears that in some cases, these genes include pathogenicity determinants.

\section{Creation of a common tongue}

Both the biosynthesis and the function of AI-2 fundamentally differ from those of every other known autoinducer (Bassler 1999; Schauder et al. 2001). As mentioned, the biosynthetic machinery responsible for production of each acyl-HSL and oligopeptide autoinducer is designed to manufacture a specific signaling molecule. The final signal molecules are related, but their slight structural differences confer species-specific communication to the systems. In contrast, in every species in which AI-2 biosynthesis has been studied, the same intermediates are generated in the biosynthetic pathway, leading to the production of signaling molecules of identical structure (Schauder et al. 2001). This facet of AI-2 biosynthesis results in a nonspecific language.

The biosynthetic pathway for AI-2 has recently been described (Schauder et al. 2001). AI-2 is generated from SAM in three enzymatic steps (Fig. 5). SAM is an essential metabolite used in central metabolism. In one if its roles, SAM is used as a methyl donor for DNA, RNA, and proteins. Many SAM-dependent methyl transferases act on SAM, and the transfer of the methyl group to a particular substrate leads to the production of $S$ adenosylhomocysteine (SAH). SAH is a potent inhibitor of SAM-dependent methyl transferases, and, therefore, must be rapidly eliminated. The nucleosidase Pfs is responsible for the detoxification step (Della Ragione et al. 1985; Cornell et al. 1996). Pfs removes adenine from $\mathrm{SAH}$ forming $S$-ribosylhomocysteine (SRH). SRH is the substrate for LuxS, and LuxS cleaves the ribosyl moiety from SRH to produce homocysteine and AI-2 (Schauder et al. 2001). Earlier analysis of this pathway showed that SRH is converted to homocysteine and 4,5-dihydroxy-2,3-pentanedione. However, the function and fate of 4,5-dihydroxy-2,3-pentanedione were not known, nor was the enzyme identified that catalyzed the formation of the pentanedione (Miller and Duerre 1968; Duerre and Walker 1977; Greene 1996). The AI-2 studies show that LuxS is this enzyme, and biochemical evidence suggests that following the LuxS catalyzed formation of 4,5-dihydroxy-2,3-pentanedione, the pentanedione spontaneously cyclizes into a furanone (Fig. 2C). The exact structure of the AI-2 furanone has not been determined, but

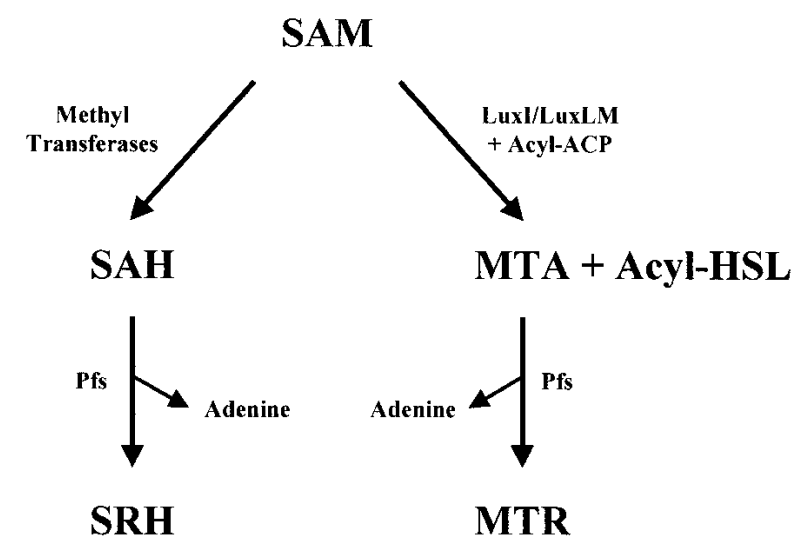

Luxs

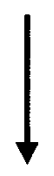

$\mathrm{AI}-2+\mathrm{HC} \quad$ ?

Figure 5. Acyl-HSL and AI-2 biosynthesis. Both AI-2 and acylHSL autoinducers are derived from $S$-adenosylmethionine (SAM). In AI-2 biosynthesis, following methyl transfer from SAM to its various substrates, $S$-adenosylhomocysteine (SAH) is formed. The Pfs enzyme removes adenine from SAH to form $S$-ribosylhomocysteine (SRH). LuxS acts on SRH to produce AI-2 and homocysteine (HC). In acyl-HSL biosynthesis, the LuxI-like enzymes (or LuxLM in $V$. harveyi) catalyze the formation of the autoinducer from SAM and a specific acyl-acyl carrier protein (acyl-ACP). Methylthioadenosine (MTA) is formed as a product of AI-1 biosynthesis. Pfs removes adenine from MTA to form methylthioribose (MTR). The further catabolism of MTR has not been characterized.

several furanones have significant AI-2 activity (Schauder et al. 2001).

The pathway shown on the left-hand side of Figure 5 has long been considered a salvage route for detoxification of SAH and for recycling of adenine and homocysteine (Greene 1996). However, these new studies show that detoxification and recycling are not the only purposes of this pathway; it is also required for synthesis of AI-2. To test the generality of this pathway, purified $E$. coli Pfs enzyme combined with LuxS enzymes purified from different bacteria were used to make AI-2 from $\mathrm{SAH}$ in vitro. This experiment was performed with LuxS protein from E. coli, S. typhimurium, V. harveyi, V. cholerae, and the Gram-positive bacterium E. facaelis. In every case, AI- 2 of identical specific activity was produced, and the other product of the LuxS reaction was homocysteine. These findings strongly suggest that, in contrast to acyl-HSL and oligopeptide autoinducers, AI-2 does not represent a family of related molecules, but rather, AI-2 from different species of bacteria is identical (Schauder et al. 2001). Perhaps bacteria require a specific, unrecognizable signal for intraspecies cell-cell communication, and a nonspecific, universally recognized signal for interspecies cell-cell communication. 


\section{Two languages are better than one}

The discovery that SAM is the starting molecule for AI-2 production is intriguing because, as mentioned, SAM is also the starting molecule for acyl-HSL biosynthesis. We have described the role of the enzyme Pfs in AI-2 biosynthesis. Additionally, Pfs has a required role in the metabolic pathway responsible for acyl-HSL production. Figure 5 shows both the AI- 2 and the acyl-HSL biosynthetic pathways. As mentioned above, in acyl-HSL biosynthesis, the LuxI enzymes (or LuxLM in $V$. harveyi) catalyze amide bond formation between a specific acylACP and SAM. When the intermediate product lactonizes to form the acyl-HSL, MTA is released (Hanzelka and Greenberg 1996; More et al. 1996; Val and Cronan 1998; Parsek et al. 1999). The intermediate product, MTA, is extremely toxic. Pfs removes adenine from MTA to form methylthioribose (MTR) (Duerre and Walker 1977; Schlenk 1983). Subsequent steps in the catabolism of MTR have not been identified in any species except $K$. pneumoniae. In K. pneumoniae, MTR is converted to methionine (Greene 1996).

These results show that bacteria that produce both an acyl-HSL autoinducer and AI-2 have linked their biosyntheses, because both processes rely on the essential cellular metabolite SAM, and also on the activity of Pfs to remove toxic byproducts of SAM utilization (Schauder et al. 2001). In order for quorum sensing to operate correctly, as bacteria grow, they must continuously produce the autoinducer signals so that their concentrations will reflect the population density. Presumably the requirement for essential substrates of intermediary metabolism guarantees an ample supply of material for signal production. Furthermore, by evolving pathways that generate toxic intermediates, the bacteria also ensure that the signal producing pathways will be driven to completion. These characteristics of autoinducer biosynthesis indicate that the production of AI-2 and acyl-HSL autoinducers is a high priority for bacteria. In Gram-positive bacteria, use of essential protein biosynthesis machinery to make autoinducers could also ensure that a constant supply of resources is available for signal generation.

No one knows why Gram-negative bacteria use acylHSLs while Gram-positive bacteria use oligopeptides for autoinducers, as the specific benefit of employing one of these types of molecules instead of the other is not understood. However, both Gram-negative and Gram-positive bacterial species have AI-2. This suggests that AI-2 may be the most ancient of the known autoinducers, and that it evolved prior to the divergence of Gram-negative and Gram-positive bacteria. Possibly, the original function of AI-2 was in the regulation of some general metabolic process(es) in the cell. This may still be the case in many or all AI-2 producing bacteria. However, in at least a few instances we now know that AI-2 also has a specialized role in cell-cell communication.

A striking feature of the AI-2 pathway is the necessity for two enzymatic steps to eliminate the toxic SAH intermediate, because eukaryotes detoxify in one step. Spe- cifically, in eukaryotes, the enzyme $S$-adenosylhomocysteinase cleaves SAH to adenosine and homocysteine (Palmer and Abeles 1979). Thus, in a single step, eukaryotes gain two usable products. However, as far as is known, eukaryotes do not make quorum sensing autoinducers. Therefore, bacteria may have evolved a more elaborate pathway than eukaryotes for detoxification because this pathway is also required for AI-2 signal production.

Finally, the AI-2 detection apparatus has not been identified in any bacterium other than $V$. harveyi. As mentioned, it has been known for nearly a decade that the LuxP protein is the primary receptor for AI-2, and LuxP is a periplasmic ribose-like binding protein (Bassler et al. 1994a). The recent AI-2 biosynthetic studies show that AI-2 is produced from the ribosyl moiety of SRH, and if AI-2 is a furanone, it closely resembles ribose. It now seems obvious that the detector for AI- 2 should resemble a sugar binding protein.

\section{Talking sense and nonsense}

Regardless of the type of autoinducer signal used, intraspecies bacterial quorum sensing allows coordinated regulation of behavior. This process enables a group of bacteria to act in a concerted manner, and thus acquire some of the characteristics of multicellular organisms. Likewise, regardless of whether AI-2 or some other general signal is used, interspecies bacterial communication can serve to synchronize the specialized functions of each species in the group. Appropriate exploitation of the diversity present in a population could enhance the survival of the entire community. Furthermore, the productive interactions promoted by quorum sensing could be instrumental in the development of multispecies bacterial organizations such as biofilms and in the establishment of specific symbiotic associations with eukaryotic hosts.

Naturally occurring pro-quorum sensing tactics have been documented in bacterial-bacterial interactions. As described above, $V$. harveyi detects AI-2 produced by other species of bacteria and uses it to regulate gene expression. We have also proposed that AI-2 is a general language that bacteria use for intergenera signaling. Another example of interspecies communication occurs in P. aeruginosa and Burkholderia cepacia biofilms. Biofilms are highly ordered structures that allow bacteria to live adhered to surfaces. Biofilms can be composed of single or multiple species, and these assemblages possess aqueous channels for distributing nutrients to the members of the community and preventing desiccation. Specialized patterns of gene expression are evident in different locations within a biofilm, indicating that the members of the community have specific duties that, in combination, enhance the survival of the entire consortium (Costerton et al. 1994, 1995). P. aeruginosa biofilms exist in the lungs of cystic fibrosis (CF) sufferers. An intact quorum sensing circuit is required for proper biofilm formation by $P$. aeruginosa (Davies et al. 1998; Parsek and Greenberg 1999|. B. cepacia is an emerging 
pathogen in CF infections (Govan et al. 1996). Usually $\mathrm{CF}$ individuals infected with $B$. cepacia are coinfected with $P$. aeruginosa (Taylor et al. 1993). Addition of $P$. aeruginosa autoinducers to $B$. cepacia induces the expression of $B$. cepacia virulence factors. It is hypothesized that $P$. aeruginosa is the primary colonizer in the CF lung, and interspecies communication allows $B$. cepacia to establish itself in the host (Lewenza et al. 1999).

Although there is no documented example, it seems probable that eukaryotic hosts in mutualistic relationships with quorum sensing bacteria elicit signals that enhance the quorum sensing capabilities of their prokaryotic partners. The animal hosts that participate in the well-studied quorum sensing partnerships are not tractable to genetic manipulation. Therefore, it has not yet been possible to ascertain whether eukaryotic to prokaryotic communication occurs that positively influences a bacterial quorum sensing circuit. Given the exquisite specificity of these partnerships, it would be astonishing if the hosts were not initiating elements of the conversation.

However, bacteria do not always live in harmony with one another or with their eukaryotic hosts. We know that, in addition to its role in the establishment of symbiotic associations, bacterial quorum sensing is also critical in the development of pathogenic relationships with eukaryotic hosts. Similarly, bacteria residing in natural environments live in intense competition with other species of bacteria for scarce resources. Therefore, evolution of antimicrobial strategies that interfere with production or perception of autoinducers could be used to thwart bacteria that depend on processes controlled by quorum sensing.

There are several examples of anti-quorum sensing strategies in use by competing populations of bacteria. As mentioned, $S$. aureus groups use peptide quorum sensing to control agr virulence and also to inhibit virulence in other S. aureus groups (Ji et al. 1997; Mayville et al. 1999; Otto et al. 1999). The plant-associated bacterium Pseudomonas aureofaciens regulates antibiotic production by an acyl-HSL quorum sensing circuit. Additionally, secreted signal molecules produced by a number of other naturally occurring species of plant-associated bacteria induce antibiotic production in $P$. aureofaciens. These findings suggest that $P$. aureofaciens detects the presence of competitor bacteria and responds by attempting to kill them (Pierson et al. 1994). An alternative strategy for preventing quorum sensing by a competitor has evolved in the soil bacterium B. subtilis. B. subtilis produces an enzyme called AiiA that appears to be a metallohydrolase. This enzyme inactivates the acyl-HSL autoinducer of Erwinia carotovora. E. carotovora is a commensal soil bacterium that uses quorum sensing to colonize plants and also to regulate antibiotic production. AiiA renders E. carotovora incapable of producing antibiotics and colonization factors (Dong et al. 2000). Presumably this strategy makes E. carotovora unable to compete effectively against $B$. subtilis. Similarly, the soil bacterium Variovorax paradoxus has been shown to be capable of using acyl-HSLs as the sole source of carbon and nitrogen. This study suggests that, in its natural habitat, $V$. paradoxus might degrade and grow on acyl-HSLs as a means to gain a competitive edge (Leadbetter and Greenberg 2000).

There is also an example of a eukaryotic strategy that has been designed to specifically interfere with bacterial quorum sensing. The seaweed Delisea pulchra produces halogenated furanones and enones that disrupt acylHSL-directed swarming motility and colonization by the pathogenic bacterium Serratia liquefaciens. Furanones are structurally related to homoserine lactones, and the furanones bind to the LuxR-like protein and prevent the autoinducer from binding (Givskov et al. 1996; Manefield et al. 1999). Note, however, that this is a model system, and D. pulchra and S. liquefaciens do not naturally encounter one another. These studies raise the possibility that true pathogens of $D$. pulchra are undermined in an analogous manner. It is especially interesting that a furanone is the inhibitor of the acyl-HSL quorum sensing system. Given the new findings that the LuxS dependent AI-2 molecule is probably a furanone, there could be cases in which AI-2 acts as an antagonist of acyl-HSL mediated quorum sensing.

Studies of bacterial and eukaryotic interference with bacterial quorum sensing are only in the beginning stages. However, the few reports that already exist suggest that, in the wild, widespread use of strategies for either helping or hindering bacterial neighbors exists. To date, autoinducer mimics, autoinducer antagonists, and autoinducer degradation systems have been reported in intergenera and inter-kingdom interactions. Many other mechanisms for enhancing and inhibiting quorum sensing can be imagined, and likely exist.

\section{Conclusions}

Studies of quorum sensing systems demonstrate that bacteria have evolved multiple languages for communicating within and between species. Intra- and interspecies cell-cell communication allows bacteria to coordinate various biological activities in order to behave like multicellular organisms. The processes controlled by quorum sensing are diverse and reflect the specific needs of particular communities inhabiting unique niches. Competing bacteria and susceptible hosts have developed natural strategies for impeding quorum sensing bacteria by destroying the chemical signal molecules or producing autoinducer antagonists that interfere with recognition of the bone fide signal molecule. Similarly, in mutualistic associations, bacteria and probably eukaryotic hosts have evolved tactics that augment the quorum sensing capabilities of beneficial bacteria. These natural therapies that disrupt/improve quorum sensing are being used as models in the design of analogous synthetic strategies intended to manipulate quorum sensing systems in bacteria. Biotechnological research is now focused on the development of molecules that are structurally related to autoinducers. Such molecules have potential use as antimicrobial drugs aimed at bacteria that use quorum sensing to control virulence. Similarly, the 
biosynthetic enzymes involved in autoinducer production and the autoinducer detection apparatuses are viewed as potential targets for novel antimicrobial drug design. Furthermore, biotechnological approaches designed to exploit beneficial quorum sensing processes could be used to improve industrial production of natural products such as antibiotics. With or without practical applications, continued study of bacterial quorum sensing systems promises to give biologists new insights into novel mechanisms of intra- and intercellular signal transmission, intra- and interspecies communication and the evolution of multicellular organisms.

\section{Acknowledgments}

The National Science Foundation grants MCB-0083160 and MCB-0094447 and The Office of Naval Research grant no. N00014-99-0767 supported this work. Dr. Schauder was supported by a DAAD (German Academic Exchange Service) fellowship.

\section{References}

Bassler, B.L. 1999. How bacteria talk to each other: Regulation of gene expression by quorum sensing. Curr. Opin. Microbiol. 2: 582-587.

Bassler, B.L., Greenberg, E.P., and Stevens, A.M. 1997. Crossspecies induction of luminescence in the quorum-sensing bacterium Vibrio harveyi. J. Bacteriol. 179: 4043-4045.

Bassler, B.L., Wright, M., Showalter, R.E., and Silverman, M.R. 1993. Intercellular signaling in Vibrio harveyi: Sequence and function of genes regulating expression of luminescence. Mol. Microbiol. 9: 773-786.

Bassler, B.L., Wright, M., and Silverman, M.R. 1994a. Multiple signaling systems controlling expression of luminescence in Vibrio harveyi: Sequence and function of genes encoding a second sensory pathway. Mol. Microbiol. 13: 273-286.

1994b. Sequence and function of LuxO, a negative regulator of luminescence in Vibrio harveyi. Mol. Microbiol. 12: 403-412.

Beck von Bodman, S., Hayman, G.T., and Farrand, S.K. 1992. Opine catabolism and conjugal transfer of the nopaline Ti plasmid pTiC58 are coordinately regulated by a single repressor. Proc. Natl. Acad. Sci. 89: 643-647.

Brint, J.M. and Ohman, D.E. 1995. Synthesis of multiple exoproducts in Pseudomonas aeruginosa is under the control of RhlR-RhlI, another set of regulators in strain PAO1 with homology to the autoinducer-responsive LuxR-LuxI family. J. Bacteriol. 177: 7155-7163.

Cao, J.G. and Meighen, E.A. 1989. Purification and structural identification of an autoinducer for the luminescence system of Vibrio harveyi. J. Biol. Chem. 264: 21670-21676.

Choi, S.H. and Greenberg, E.P. 1991. The C-terminal region of the Vibrio fischeri LuxR protein contains an inducer-independent lux gene activating domain. Proc. Natl. Acad. Sci. 88: $11115-1119$.

-1992. Genetic dissection of DNA binding and luminescence gene activation by the Vibrio fischeri LuxR protein. J. Bacteriol. 174: 4064-4069.

Christie, P.J. 1997. Agrobacterium tumefaciens T-complex transport apparatus: A paradigm for a new family of multifunctional transporters in eubacteria. J. Bacteriol. 179: 30853094.
Chugani, S.A., Whiteley, M., Lee, K.M., D'Argenio, D., Manoil, C., and Greenberg, E.P. 2001. QscR, a modulator of quorumsensing signal synthesis and virulence in Pseudomonas aeruginosa. Proc. Nat1. Acad. Sci. 98: 2752-2757.

Cornell, K.A., Swarts, W.E., Barry, R.D., and Riscoe, M.K. 1996. Characterization of recombinant Eschericha coli 5' - methylthioadenosine/S-adenosylhomocysteine nucleosidase: Analysis of enzymatic activity and substrate specificity. Biochem. Biophys. Res. Commun. 228: 724-732.

Costerton, J.W., Lewandowski, Z., Caldwell, D.E., Korber, D.R., and Lappin-Scott, H.M. 1995. Microbial biofilms. Annu. Rev. Microbiol. 49: 711-745.

Costerton, J.W., Lewandowski, Z., DeBeer, D., Caldwell, D., Korber, D., and James, G. 1994. Biofilms, the customized microniche. J. Bacteriol. 176: 2137-2142.

Davies, D.G., Parsek, M.R., Pearson, J.P., Iglewski, B.H., Costerton, J.W., and Greenberg, E.P. 1998. The involvement of cellto-cell signals in the development of a bacterial biofilm. Science 280: 295-298.

Day, W.A. and Maurelli, A.T. 2001. Shigella flexneri LuxS quorum-sensing system modulates virB expression but is not essential for virulence. Infect. Immunol. 69: 15-23.

de Kievit, T.R. and Iglewski, B.H. 2000. Bacterial quorum sensing in pathogenic relationships. Infect. Immunol. 68: 48394849.

Della Ragione, F., Porcelli, M., Carteni-Farina, M., Zappia, V., and Pegg, A.E. 1985. Escherichia coli S-adenosylhomocysteine $/ 5^{\prime}$-methylthioadenosine nucleosidase. Purification, substrate specificity and mechanism of action. Biochem. J. 232: 335-341.

Dessaux, Y., Petit, A., and Tempe, J. 1992. Opines in Agrobacterium Biology. In Molecular Signals in Plant-Microbe Interactions (ed. D.P.S. Verma), pp. 109-136. CRC Press, Boca Raton, FL.

Dong, Y.H., Xu, J.L., Li, X.Z., and Zhang, L.H. 2000. AiiA, an enzyme that inactivates the acylhomoserine lactone quorum-sensing signal and attenuates the virulence of Erwinia carotovora. Proc. Natl. Acad. Sci. 97: 3526-3531.

Duerre, J.A. and Walker, R.D. 1977. Metabolism of adenosylhomocysteine. In The Biochemistry of Adenosylmethionine (eds. F. Salvatore, E. Borek, V. Zappia, H.G. Williams-Ashman, and F. Schlenk), pp. 43-57. Columbia University Press, New York.

Engebrecht, J., Nealson, K., and Silverman, M. 1983. Bacterial bioluminescence: Isolation and genetic analysis of functions from Vibrio fischeri. Cell 32: 773-781.

Engebrecht, J. and Silverman, M. 1984. Identification of genes and gene products necessary for bacterial bioluminescence. Proc. Natl. Acad. Sci. 81: 4154-4158.

. 1987. Nucleotide sequence of the regulatory locus controlling expression of bacterial genes for bioluminescence. Nucleic Acids Res. 15: 10455-10467.

Freeman, J.A. and Bassler, B.L. 1999a. A genetic analysis of the function of LuxO, a two-component response regulator involved in quorum sensing in Vibrio harveyi. Mol. Microbiol. 31: 665-677.

- 1999b. Sequence and function of LuxU: A two-component phosphorelay protein that regulates quorum sensing in Vibrio harveyi. J. Bacteriol. 181: 899-906.

Freeman, J.A., Lilley, B.N., and Bassler, B.L. 2000. A genetic analysis of the functions of LuxN: A two-component hybrid sensor kinase that regulates quorum sensing in Vibrio harveyi. Mol. Microbiol. 35: 139-149.

Fuqua, C., Burbea, M., and Winans, S.C. 1995. Activity of the Agrobacterium Ti plasmid conjugal transfer regulator TraR is inhibited by the product of the traM gene. J. Bacteriol. 
177: $1367-1373$.

Fuqua, C. and Winans, S.C. 1996. Localization of OccR-activated and TraR-activated promoters that express two ABCtype permeases and the traR gene of Ti plasmid pTiR10. Mol. Microbiol. 20: 1199-1210.

Fuqua, C., Winans, S.C., and Greenberg, E.P. 1996. Census and consensus in bacterial ecosystems: The LuxR-LuxI family of quorum-sensing transcriptional regulators. Annu. Rev. Microbiol. 50: 727-751.

Fuqua, W.C. and Winans, S.C. 1994. A LuxR-LuxI type regulatory system activates Agrobacterium Ti plasmid conjugal transfer in the presence of a plant tumor metabolite. J. Bacteriol. 176: 2796-2806.

Givskov, M., de Nys, R., Manefield, M., Gram, L., Maximilien, R., Eberl, L., Molin, S., Steinberg, P.D., and Kjelleberg, S. 1996. Eukaryotic interference with homoserine lactone-mediated prokaryotic signaling. J. Bacteriol. 178: 6618-6622.

Govan, J.R., Hughes, J.E., and Vandamme, P. 1996. Burkholderia cepacia: Medical, taxonomic and ecological issues. I. Med. Microbiol. 45: 395-407.

Gray, K.M., Passador, L., Iglewski, B.H., and Greenberg, E.P. 1994. Interchangeability and specificity of components from the quorum-sensing regulatory systems of Vibrio fischeri and Pseudomonas aeruginosa. J. Bacteriol. 176: 3076-3080.

Greene, R.C. 1996. Biosynthesis of Methionine. In Escherichia coli and Salmonella: Cellular and Molecular Biology (ed. F. Neidhardt). ASM Press, Washington, DC.

Grossman, A.D. 1995. Genetic networks controlling the initiation of sporulation and the development of genetic competence in Bacillus subtilis. Annu. Rev. Genet. 29: 477-508.

Hanzelka, B.L. and Greenberg, E.P. 1996. Quorum sensing in Vibrio fischeri: Evidence that $S$-adenosylmethionine is the amino acid substrate for autoinducer synthesis. J. Bacteriol. 178: 5291-5294.

Hanzelka, B.L., Parsek, M.R., Val, D.L., Dunlap, P.V., Cronan, Jr., J.E., and Greenberg, E.P. 1999. Acylhomoserine lactone synthase activity of the Vibrio fischeri AinS protein. I. Bacteriol. 181: 5766-5770.

Hassett, D.J., Ma, J.F., Elkins, J.G., McDermott, T.R., Ochsner, U.A., West, S.E., Huang, C.T., Fredericks, J., Burnett, S., Stewart, P.S., et al. 1999. Quorum sensing in Pseudomonas aeruginosa controls expression of catalase and superoxide dismutase genes and mediates biofilm susceptibility to hydrogen peroxide. Mol. Microbiol. 34: 1082-1093.

Hastings, J.W. and Nealson, K.H. 1977. Bacterial bioluminescence. Annu. Rev. Microbiol. 31: 549-595.

Hoch, J.A. 1995. Control of cellular development in sporulating bacteria by the phosphorelay two-component signal transduction system. In Two-Component Signal Transduction (eds. J.A. Hoch and T.J. Silhavy), pp. 129-144. ASM Press, Washington, DC.

Hwang, I., Li, P.L., Zhang, L., Piper, K.R., Cook, D.M., Tate, M.E., and Farrand, S.K. 1994. TraI, a LuxI homologue, is responsible for production of conjugation factor, the Ti plasmid N-acylhomoserine lactone autoinducer. Proc. Natl. Acad. Sci. 91: 4639-4643.

Ji, G., Beavis, R.C., and Novick, R.P. 1995. Cell density control of staphylococcal virulence mediated by an octapeptide pheromone. Proc. Natl. Acad. Sci. 92: 12055-12059.

. 1997. Bacterial interference caused by autoinducing peptide variants. Science 276: 2027-2030.

Jones, S., Yu, B., Bainton, N.J., Birdsall, M., Bycroft, B.W., Chhabra, S.R., Cox, A.J., Golby, P., Reeves, P.J., Stephens, S., et al. 1993. The lux autoinducer regulates the production of exoenzyme virulence determinants in Erwinia carotovora and Pseudomonas aeruginosa. EMBO J. 12: 2477-2482.
Joyce, E.A., Bassler, B.L., and Wright, A. 2000. Evidence for a signaling system in Helicobacter pylori: Detection of a luxSencoded autoinducer. J. Bacteriol. 182: 3638-3643.

Kaplan, H.B. and Greenberg, E.P. 1985. Diffusion of autoinducer is involved in regulation of the Vibrio fischeri luminescence system. J. Bacteriol. 163: 1210-1214.

Kim, S.Y., Lee, S.E., Kim, Y.R., Kim, J.H., Ryu, P.Y., Chung, S.S., and Rhee, J.H. 2000. Virulence regulatory role of luxS quorum sensing system in Vibrio vulmificus. In ASM General Meeting, pp. Abstract B-248.

Kleerebezem, M., Quadri, L.E., Kuipers, O.P., and de Vos, W.M. 1997. Quorum sensing by peptide pheromones and two-component signal-transduction systems in Gram-positive bacteria. Mol. Microbiol. 24: 895-904.

Latifi, A., Foglino, M., Tanaka, K., Williams, P., and Lazdunski, A. 1996. A hierarchical quorum-sensing cascade in Pseudomonas aeruginosa links the transcriptional activators LasR and RhIR (VsmR) to expression of the stationary-phase sigma factor RpoS. Mol. Microbiol. 21: 1137-1146.

Lazazzera, B.A. and Grossman, A.D. 1998. The ins and outs of peptide signaling. Trends Microbiol. 6: 288-294.

Lazazzera, B.A., Solomon, J.M., and Grossman, A.D. 1997. An exported peptide functions intracellularly to contribute to cell density signaling in B. subtilis. Cell 89: 917-925.

Leadbetter, J.R. and Greenberg, E.P. 2000. Metabolism of acylhomoserine lactone quorum-sensing signals by Variovorax paradoxus. J. Bacteriol. 182: 6921-6926.

Lewenza, S., Conway, B., Greenberg, E.P., and Sokol, P.A. 1999. Quorum sensing in Burkholderia cepacia: Identification of the LuxRI homologs CepRI. J. Bacteriol. 181: 748-756.

Lilley, B.N. and Bassler, B.L. 2000. Regulation of quorum sensing in Vibrio harveyi by LuxO and sigma-54. Mol. Microbiol. 36: 940-954.

Luo, Z.Q., Qin, Y., and Farrand, S.K. 2000. The antiactivator TraM interferes with the autoinducer-dependent binding of TraR to DNA by interacting with the C-terminal region of the quorum-sensing activator. J. Biol. Chem. 275: 77137722.

Lyon, W.R., Madden, J.C., Stein, J., and Caparon, M.G. 2000. Mutation of luxS affects growth and virulence factor expression in Streptococcus pyogenes. Mol. Microbiol. (in press).

Magnuson, R., Solomon, J., and Grossman, A.D. 1994. Biochemical and genetic characterization of a competence pheromone from B. subtilis. Cell 77: 207-216.

Manefield, M., de Nys, R., Kumar, N., Read, R., Givskov, M., Steinberg, P., and Kjelleberg, S. 1999. Evidence that halogenated furanones from Delisea pulchra inhibit acylated homoserine lactone (AHL)-mediated gene expression by displacing the AHL signal from its receptor protein. Microbiology 145: 283-291.

Martin, M., Showalter, R., and Silverman, M. 1989. Identification of a locus controlling expression of luminescence genes in Vibrio harveyi. J. Bacteriol. 171: 2406-2414.

Mayville, P., Ji, G., Beavis, R., Yang, H., Goger, M., Novick, R.P., and Muir, T.W. 1999. Structure-activity analysis of synthetic autoinducing thiolactone peptides from Staphylococcus aureus responsible for virulence. Proc. Natl. Acad. Sci. 96: $1218-1223$.

Miller, C.H. and Duerre, J.A. 1968. S-ribosylhomocysteine cleavage enzyme from Escherichia coli. J. Biol. Chem. 243: 92-97.

Miller, M.B. and Bassler, B.L. 2001. Quorum Sensing in Bacteria. Annu. Rev. Microbiol. 55: 165-199.

More, M.I., Finger, L.D., Stryker, J.L., Fuqua, C., Eberhard, A., and Winans, S.C. 1996. Enzymatic synthesis of a quorumsensing autoinducer through use of defined substrates. Sci- 
ence 272: 1655-1658.

Morfeldt, E., Janzon, L., Arvidson, S., and Lofdahl, S. 1988. Cloning of a chromosomal locus (exp) which regulates the expression of several exoprotein genes in Staphylococcus aureus. Mol. Gen. Genet. 211: 435-440.

Morfeldt, E., Taylor, D., von Gabain, A., and Arvidson, S. 1995. Activation of alpha-toxin translation in Staphylococcus aureus by the trans-encoded antisense RNA, RNAIII. EMBO $J$. 14: 4569-4577.

Nealson, K.H. and Hastings, J.W. 1979. Bacterial bioluminescence: Its control and ecological significance. Microbiol. Rev. 43: 496-518.

Novick, R.P. 1999. Regulation of pathogenicity in Staphylococcus aureus by a peptide-based density-sensing system. In Cell-Cell Signaling in Bacteria (eds. G.M. Dunney and S.C. Winans), pp. 129-146. ASM Press, Washington, DC.

Novick, R.P., Projan, S.J., Kornblum, J., Ross, H.F., Ji, G., Kreiswirth, B., Vandenesch, F., and Moghazeh, S. 1995. The agr P2 operon: An autocatalytic sensory transduction system in Staphylococcus aureus. Mol. Gen. Genet. 248: 446-458.

Ochsner, U.A. and Reiser, J. 1995. Autoinducer-mediated regulation of rhamnolipid biosurfactant synthesis in Pseudomonas aeruginosa. Proc. Nat1. Acad. Sci. 92: 6424-6428.

Otto, M., Sussmuth, R., Vuong, C., Jung, G., and Gotz, F. 1999. Inhibition of virulence factor expression in Staphylococcus aureus by the Staphylococcus epidermidis agr pheromone and derivatives. FEBS Lett. 450: 257-262.

Palmer, J.L. and Abeles, R.H. 1979. The mechanism of action of S-adenosylhomocysteinase. J. Biol. Chem. 254: 1217-1226.

Parsek, M.R. and Greenberg, E.P. 1999. Quorum sensing signals in development of Pseudomonas aeruginosa biofilms. Methods Enzymol. 310: 43-55.

Parsek, M.R., Val, D.L., Hanzelka, B.L., Cronan, Jr., J.E., and Greenberg, E.P. 1999. Acyl homoserine-lactone quorumsensing signal generation. Proc. Natl. Acad. Sci. 96: 43604365.

Passador, L., Cook, J.M., Gambello, M.J., Rust, L., and Iglewski, B.H. 1993. Expression of Pseudomonas aeruginosa virulence genes requires cell-to-cell communication. Science 260: $1127-1130$

Pearson, J.P., Pesci, E.C., and Iglewski, B.H. 1997. Roles of Pseudomonas aeruginosa las and rhl quorum-sensing systems in control of elastase and rhamnolipid biosynthesis genes. $J$. Bacteriol. 179: 5756-5767.

Peng, H.L., Novick, R.P., Kreiswirth, B., Kornblum, J., and Schlievert, P. 1988. Cloning, characterization, and sequencing of an accessory gene regulator $(a g r)$ in Staphylococcus aureus. J. Bacteriol. 170: 4365-4372.

Perego, M. 1997. A peptide export-import control circuit modulating bacterial development regulates protein phosphatases of the phosphorelay. Proc. Nat1. Acad. Sci. 94: 8612-8617.

Perego, M., Hanstein, C., Welsh, K.M., Djavakhishvili, T., Glaser, P., and Hoch, J.A. 1994. Multiple protein-aspartate phosphatases provide a mechanism for the integration of diverse signals in the control of development in B. subtilis. Cell 79: 1047-1055.

Pesci, E.C., Milbank, J.B., Pearson, J.P., McKnight, S., Kende, A.S., Greenberg, E.P., and Iglewski, B.H. 1999. Quinolone signaling in the cell-to-cell communication system of Pseudomonas aeruginosa. Proc. Natl. Acad. Sci. 96: 1122911234.

Pesci, E.C., Pearson, J.P., Seed, P.C., and Iglewski, B.H. 1997. Regulation of las and $\mathrm{rhl}$ quorum sensing in Pseudomonas aeruginosa. J. Bacteriol. 179: 3127-3132.

Pierson, L.S., III, Keppenne, V.D., and Wood, D.W. 1994. Phenazine antibiotic biosynthesis in Pseudomonas aureofaciens
30-84 is regulated by $\mathrm{PhzR}$ in response to cell density. J. Bacteriol. 176: 3966-3974.

Piper, K.R., Beck von Bodman, S., and Farrand, S.K. 1993. Conjugation factor of Agrobacterium tumefaciens regulates $\mathrm{Ti}$ plasmid transfer by autoinduction. Nature 362: 448-450.

Poellinger, K.A., Lee, J.P., Parales, Jr., J.V., and Greenberg, E.P. 1995. Intragenic suppression of a luxR mutation: Characterization of an autoinducer-independent LuxR. FEMS Microbiol. Lett. 129: 97-101.

Ruby, E.G. 1996. Lessons from a cooperative, bacterial-animal association: The Vibrio fischeri-Euprymna scolopes light organ symbiosis. Annu. Rev. Microbiol. 50: 591-624.

Schauder, S., Shokat, K., Surette, M.G., and Bassler, B.L. 2001. The LuxS-family of bacterial autoinducers: Biosynthesis of a novel quorum sensing signal molecule. Mol. Microbiol. (in press).

Schlenk, F. 1983. Methylthioadenosine. Adv. Enzymol. Relat. Areas Mol. Biol. 54: 195-265.

Sheng, J. and Citovsky, V. 1996. Agrobacterium-plant cell DNA transport: Have virulence proteins, will travel. Plant Cell 8: $1699-1710$.

Showalter, R.E., Martin, M.O., and Silverman, M.R. 1990. Cloning and nucleotide sequence of $l u x R$, a regulatory gene controlling bioluminescence in Vibrio harveyi. J. Bacteriol. 172: 2946-2954.

Slock, J., VanRiet, D., Kolibachuk, D., and Greenberg, E.P. 1990. Critical regions of the Vibrio fischeri LuxR protein defined by mutational analysis. I. Bacteriol. 172: 3974-3979.

Solomon, J.M., Lazazzera, B.A., and Grossman, A.D. 1996. Purification and characterization of an extracellular peptide factor that affects two different developmental pathways in Bacillus subtilis. Genes \& Dev. 10: 2014-2024.

Solomon, J.M., Magnuson, R., Srivastava, A., and Grossman, A.D. 1995. Convergent sensing pathways mediate response to two extracellular competence factors in Bacillus subtilis. Genes, \& Dev. 9: 547-558.

Sperandio, V., Mellies, J.L., Nguyen, W., Shin, S., and Kaper, J.B. 1999. Quorum sensing controls expression of the type III secretion gene transcription and protein secretion in enterohemorrhagic and enteropathogenic Escherichia coli. Proc. Nat1. Acad. Sci. 96: 15196-15201.

Stevens, A.M., Dolan, K.M., and Greenberg, E.P. 1994. Synergistic binding of the Vibrio fischeri LuxR transcriptional activator domain and RNA polymerase to the lux promoter region. Proc. Nat1. Acad. Sci. 91: 12619-12623.

Stevens, A.M., Fujita, N., Ishihama, A., and Greenberg, E.P. 1999. Involvement of the RNA polymerase alpha-subunit $\mathrm{C}$-terminal domain in LuxR-dependent activation of the $\mathrm{Vib}$ rio fischeri luminescence genes. I. Bacteriol. 181: 47044707.

Stevens, A.M. and Greenberg, E.P. 1997. Quorum sensing in Vibrio fischeri: Essential elements for activation of the luminescence genes. J. Bacteriol. 179: 557-562.

Surette, M.G. and Bassler, B.L. 1998. Quorum sensing in Escherichia coli and Salmonella typhimurium. Proc. Natl. Acad. Sci. 95: 7046-7050.

Surette, M.G., Miller, M.B., and Bassler, B.L. 1999. Quorum sensing in Escherichia coli, Salmonella typhimurium, and Vibrio harveyi: A new family of genes responsible for autoinducer production. Proc. Natl. Acad. Sci. 96: 1639-1644.

Taylor, R.F., Gaya, H., and Hodson, M.E. 1993. Pseudomonas cepacia: Pulmonary infection in patients with cystic fibrosis. Respir. Med. 87: 187-192.

Turgay, K., Hahn, J., Burghoorn, J., and Dubnau, D. 1998. Competence in Bacillus subtilis is controlled by regulated proteolysis of a transcription factor. EMBO J. 17: 6730-6738. 
Turgay, K., Hamoen, L.W., Venema, G., and Dubnau, D. 1997. Biochemical characterization of a molecular switch involving the heat shock protein $\mathrm{ClpC}$, which controls the activity of ComK, the competence transcription factor of Bacillus subtilis. Genes \& Dev. 11: 119-128.

Val, D.L. and Cronan, Jr., J.E. 1998. In vivo evidence that $S$ adenosylmethionine and fatty acid synthesis intermediates are the substrates for the LuxI family of autoinducer synthases. J. Bacteriol. 180: 2644-2651.

van Sinderen, D., Luttinger, A., Kong, L., Dubnau, D., Venema, G., and Hamoen, L. 1995. comK encodes the competence transcription factor, the key regulatory protein for competence development in Bacillus subtilis. Mol. Microbiol. 15: 455-462.

Visick, K.L. and McFall-Ngai, M.J. 2000. An exclusive contract: Specificity in the Vibrio fischeri-Euprymna scolopes partnership. J. Bacteriol. 182: 1779-1787.

Whiteley, M., Lee, K.M., and Greenberg, E.P. 1999. Identification of genes controlled by quorum sensing in Pseudomonas aeruginosa. Proc. Natl. Acad. Sci. 96: 13904-13909.

Zhang, L., Murphy, P.J., Kerr, A., and Tate, M.E. 1993. Agrobacterium conjugation and gene regulation by N-acyl-L-homoserine lactones. Nature 362: 446-448. 




\section{The languages of bacteria}

Stephan Schauder and Bonnie L. Bassler

Genes Dev. 2001, 15:

Access the most recent version at doi:10.1101/gad.899601

References This article cites 99 articles, 61 of which can be accessed free at: http://genesdev.cshlp.org/content/15/12/1468.full.html\#ref-list-1

License

Email Alerting Receive free email alerts when new articles cite this article - sign up in the box at the top Service right corner of the article or click here.



